\title{
Fast MRI Methods for the Clinical Evaluation of Skeletal Disorders
}

\author{
Renato Toffanin ${ }^{1}$, Giuseppe Guglielmi ${ }^{2,3}$ and Maria A. Cova ${ }^{4}$ \\ ${ }^{1}$ Advanced Research Centre for Health, Environment and Space (ARCHES) \\ ${ }^{2}$ Dept. of Radiology, Casa Sollievo della Sofferenza, IRCCS \\ ${ }^{3}$ Dept. of Radiology, University of Foggia \\ ${ }^{4}$ Dept. of Radiology, University of Trieste \\ Italy
}

\section{Introduction}

Evaluation of specific magnetic resonance (MR) parameters of the skeletal system holds great potential for the accurate clinical assessment of degenerative changes occurring in bone and soft tissues at different anatomical sites. MR imaging of the water in a joint can provide anatomical information about all the soft tissues within the synovial sac (articular cartilage, meniscus, ligaments, synovial fluid) and surrounding it (muscles, tendons, vascular structures). Importantly too, MRI of the water-plus-fat can provide information pertaining both to the bone density and trabecular architecture. Recent developments have led to combinations of scan protocols and image-measurement software such that MRI can be used to evaluate the spatial distribution of specific relaxation parameters and thus detect, assess, and quantify the many pathologic processes affecting the skeletal tissues. In the articular cartilage of knee, for example, gradual deterioration of the chondral tissue leads to progressive increases in the transverse relaxation time (T2) of the water protons (David-Vaudey, 2004; Dunn et al., 2004; Apprich et al., 2010). Similarly, bone loss in the calcaneus of patients with varying degrees of osteopenia and osteoporosis causes a prolongation of the effective transverse relaxation time $\left(\mathrm{T}_{2}^{*}\right)$ of the bone marrow protons (Wehrli et al., 1995; Damilakis et al, 2004). Nonetheless, standard scan protocols for quantitative MRI are relatively slow and, therefore, not suitable for routine clinical applications. Faster methods would highly enhance their applicability in the clinical evaluation of skeletal disorders.

The purpose of this chapter is to provide a perspective on fast MRI methods for the noninvasive assessment of the skeletal status and their relevance to the diagnosis of osteoporosis and osteoarthritis, two major public health burdens (Hannan et al., 2001; Theis et al., 2007). The emphasis lies on echo-planar imaging (EPI)-based sequences (Tsao, 2010) for the accurate evaluation of pathologic processes affecting bone marrow and cartilage at different anatomical locations. A multi-shot EPI sequence is proposed for the fast $\mathrm{T}^{*}$ mapping of the lumbar bone marrow while a gradient- and spin-echo (GRASE) sequence is suggested for the fast T2 mapping of patellar articular cartilage. The description of these fast acquisition techniques is followed by a presentation of two in 
vivo feasibility studies on a clinical 1.5 T MRI scanner. These investigations demonstrate that the proposed MRI methods can produce relaxation maps of specific skeletal sites in just a few minutes, and with mean values comparable with data obtained using conventional sequences. Their potential application in the clinical evaluation of osteoporosis and osteoarthritis is also discussed.

\section{Fast MRI techniques}

Transverse relaxation is the result of random interactions at the atomic and molecular levels (Abragam, 1961). This physical phenomenon is primarily related to the intrinsic field caused by adjacent protons (spins) and hence is called spin-spin relaxation. Transverse relaxation causes irreversible dephasing of the transverse magnetization. There is also a reversible bulk field dephasing effect caused by local field inhomogeneities, and its characteristic time is referred to as $\mathrm{T}_{2}^{*}$ relaxation. These additional dephasing fields come from the main magnetic field inhomogeneity, the differences in magnetic susceptibility among various tissues or materials, chemical shift, and gradients applied for spatial encoding (Mugler, 2006). This dephasing can be eliminated by using a $180^{\circ}$ pulse, as in a spin-echo sequence. Therefore, in a spin-echo sequence, only the 'true' T2 relaxation is seen. In gradient-echo sequences, there is no $180^{\circ}$ refocusing pulse, and these dephasing effects are not eliminated. Hence, transverse relaxation in gradient-echo sequences (i.e., $\mathrm{T}^{*}$ relaxation) is a combination of 'true' $\mathrm{T} 2$ relaxation and relaxation caused by magnetic field inhomogeneities.

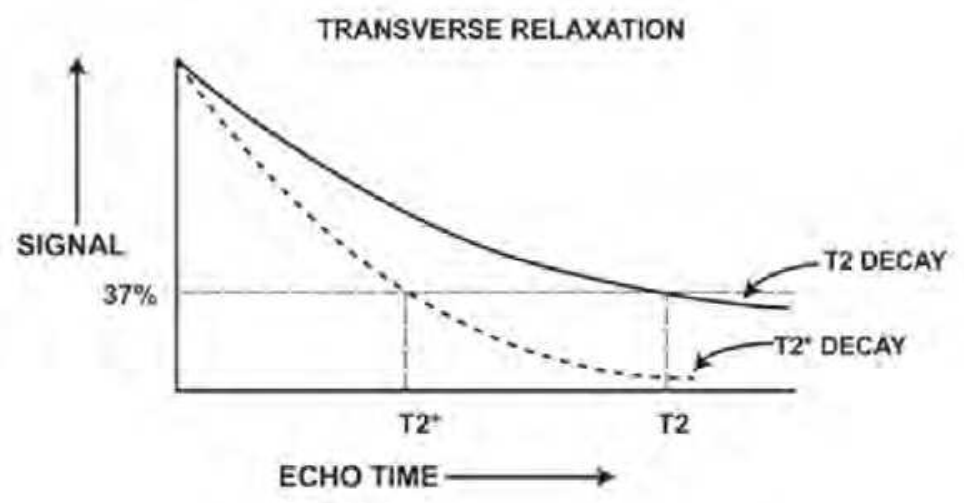

Fig. 1. A diagram showing the $\mathrm{T} 2$ and $\mathrm{T} 2 *$ relaxation decay curves

In order to obtain an accurate estimate of the transverse relaxation decay curves several images obtained at different echo times are generally required. The gold standard for T2 acquisition is likely to be a single slice single echo sequence (i.e. spin-echo sequence), repeated at several echo times, with long TR. A major improvement of the spin-echo technique is represented by the turbo spin-echo (TSE) sequence, of which a simplified diagram is depicted in Fig. 2. 


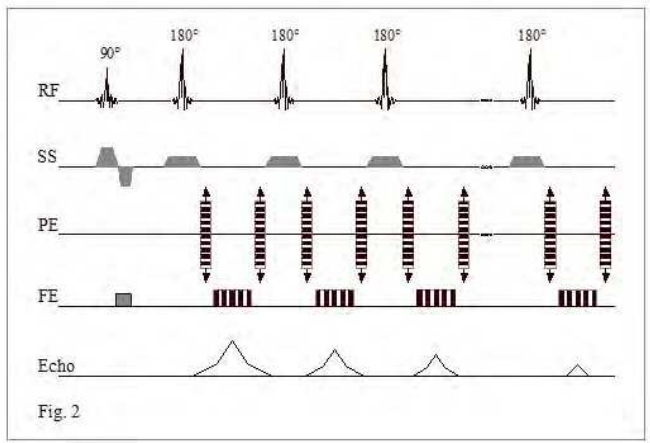

Fig. 2. A simplified diagram for the turbo spin-echo pulse sequence. RF: radiofrequency pulse, SS: slice selection gradient, PE: phase encoding gradient, FE: frequency encoding gradient

This sequence is based on multi-echo multi-shot (MEMS) (Mehlkopf et al., 1984) and rapid acquisition with relaxation enhancement (RARE) (Hennig et al., 1986) sequences and provides T2-weighted images at fractions of the acquisition time of the conventional spinecho images. By applying multiple refocusing $180^{\circ} \mathrm{RF}$ pulses after the first echo, additional spin echoes can be generated. Between each successive echo, the phase-encoding gradients can be used to prepare the spins for different lines in k-space. Thus, multiple lines in k-space can be sampled per excitation. Each echo in the readout train is progressively weaker, as defined by the T2 decay.

Another method of decreasing image acquisition time is by echo-planar imaging (EPI) (Mansfield, 1977). In EPI multiple lines of k-space are acquired through a multiple-echo readout. However, in EPI signals are produced by rapid switching of gradient polarity in place of the slower selective $180^{\circ} \mathrm{RF}$ pulses. In this way, EPI can produce an image in less than $100 \mathrm{~ms}$. However, in EPI sequences, since the multiple echoes are refocused by gradients and not by $180^{\circ}$ pulses, there is more effect of $\mathrm{T}^{*}$ decay and other artefacts. Therefore, both spin-echo and gradient-echo EPI sequences may be applied for the fast evaluation of the $\mathrm{T} 2 *$ relaxation.

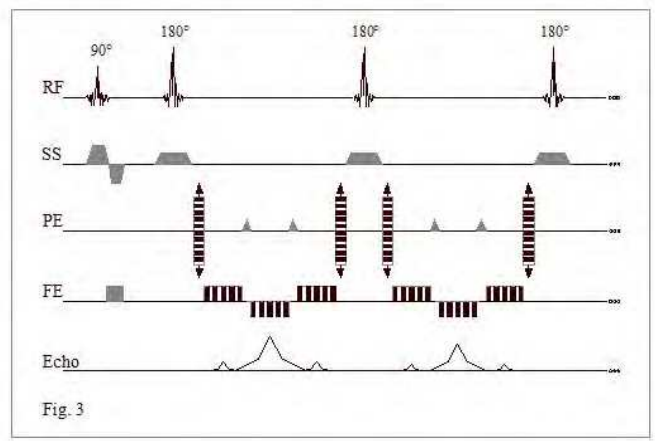

Fig. 3. A simplified diagram for the GRASE (gradient- and spin-echo) pulse sequence 
By combining the TSE and EPI methods, the GRASE (gradient- and spin-echo) sequence (Fig. 3) uses a train of refocusing $180^{\circ}$ pulses, but for each spin-echo of the readout, there are additional gradient recall echoes (Feinberg \& Oshio, 1991; Oshio \& Feinberg, 1991). In this sequence, each successive spin-echo is progressively weaker, as defined by T2 decay whereas the strength of the gradient recalled echoes surrounding the spin-echo is defined by the T2* decay envelope. By combining spin-echoes and short gradient-echo trains, the GRASE technique overcomes several potential problems of EPI, including large chemical shift, image distortions and signal loss from field inhomogeneity.

\section{MRI of trabecular bone}

Even though bone cannot be evaluated with most of the available MRI techniques in that they are unable to generate sufficient signal, new quantitative MRI approaches are used to study trabecular bone density and structure (Wehrli et al., 2006; Majumdar, 2008). MRI can be used to evaluate trabecular bone in a number of skeletal sites, indirectly via the protons of the bone marrow. Indeed, the presence of the trabecular bone matrix affects the signal intensity of bone marrow, an effect that is particularly pronounced with certain MRI sequences. With respect to gradient-echo acquisitions, static magnetic field inhomogeneities produced by the difference between trabecular bone and neighbouring bone marrow cause a more rapid decay of the MRI signal, which can be quantified by measuring T2*. Pioneering studies have shown that T2* is correlated with trabecular bone density (Davis et al., 1986; Rosenthal et al., 1990), and therefore, the effective transverse relaxation $\left(\mathrm{T} 2^{*}\right)$ is shorter in normal trabecular bone than in the less dense trabecular structures of osteoporotic bone tissue. It has also been shown that bone marrow $\mathrm{T} 2$ * reflects the orientation of the trabeculae and correlates with their mechanical strength (Chung et al., 1993; Jergas et al., 1995). These characteristics make MRI a fundamental tool in evaluating the quality of spongy bone and increase the ability of the technique not only in identifying occult fractures but also in making possible a more accurate prediction of fracture risk.

T2* relaxometry has been conducted at several sites of both axial and peripheral skeleton (Funke et al., 1994; Grampp et al., 1995; Link et al., 1998). The preferred site for quantitative MRI studies is the calcaneus in that it is mostly composed of spongy bone (95\%). Therefore quantitative MRI of the calcaneus is extremely sensitive in identifying changes in bone quality that are not revealed by bone mineral densitometry. In one MRI study at $1.5 \mathrm{~T}$ that examined 68 women with different degrees of vertebral deformity (Wehrli et al., 2002), it was demonstrated that of the various areas of the calcaneus examined, the subtalar region was best able to discriminate patients with fracture from those without. The authors of this study also demonstrated that the $\mathrm{R} 2^{*}\left(1 / \mathrm{T} 2^{*}\right)$ is sensitive to changes in bone quality that were not identified with BMD.

Trabecular bone is also prominent in the vertebral body (up to 90\%). The spine certainly represents the most critical skeletal site for quantitative MRI since vertebral fractures are the most common type of osteoporotic fractures (Wasnisch, 1999). In one MRI investigation done at $1.5 \mathrm{~T}$ on a group of 54 postmenopausal women, $\mathrm{T} 2^{*}$ mapping of the lumbar spine was shown to be capable of differentiating between healthy subjects and subjects with low energy fractures (Damilakis et al., 2004). 
The use of $\mathrm{T}^{*}$ relaxometry can certainly promote the application of quantitative MRI in the diagnosis of osteoporosis. Nonetheless, MRI protocols commonly applied to estimate $\mathrm{T} 2 *$ in bone marrow are relatively slow and, therefore, not suitable for routine clinical application. In one recent study on the calcaneus of six healthy volunteers, Toffanin et al. (2006) demonstrated the possibility of ultrafast T2* mapping of the bone marrow both at 1.5 and $3 \mathrm{~T}$. To obtain an accurate estimate of $\mathrm{T}^{*}$ at $3.0 \mathrm{~T}$ or higher magnetic fields, corrective measures may be required during postprocessing to minimise local field variations $(\Delta \mathrm{B} 0)$ responsible for signal loss and consequent overestimation of the R2* relaxation rate $\left(1 / \mathrm{T} 2^{*}\right)$. In the method proposed by Dahnke and Schaeffter (2005), the main field heterogeneity is derived from $\mathrm{T}^{*}$ calculated on more than one slice and is used as an initial value for interactive optimisation, with which the relaxation signal is corrected for each voxel.

\subsection{Fast T2* mapping of the lumbar bone marrow}

The feasibility of a multi-shot gradient-echo EPI sequence for the fast $\mathrm{T}^{*}$ mapping of the lumbar bone marrow was evaluated by our research team on a commercial clinical $1.5 \mathrm{~T}$ MRI scanner located in the Department of Radiology of the Cattinara Hospital at the University of Trieste. The MRI trial was performed on 21 subjects ( 8 males and 13 females) referred to the hospital for low back pain. Five slices were acquired to image the lumbar spine in the sagittal plane and the L2 vertebral body in the axial plane. In both cases, a fast field-echo (FFE) multi-shot EPI sequence was applied with removed blip gradients in order to apply the same phase encoding to all gradient echoes The overall examination time was approximately 5 minutes. The main acquisition parameters are summarised in Table 1.

\begin{tabular}{|l|c|c|}
\hline & Sagittal plane & Axial plane \\
\hline TR & $400 \mathrm{~ms}$ & $400 \mathrm{~ms}$ \\
\hline TEmin & $2.0 \mathrm{~ms}$ & $2.0 \mathrm{~ms}$ \\
\hline TEmax & $15.8 \mathrm{~ms}$ & $15.8 \mathrm{~ms}$ \\
\hline EPI factor & 25 & 25 \\
\hline Flip angle & $30^{\circ}$ & $30^{\circ}$ \\
\hline FOV & $300 \mathrm{~mm} \times 300 \mathrm{~mm}$ & $200 \mathrm{~mm} \times 200 \mathrm{~mm}$ \\
\hline Matrix & $320 \times 320$ & $224 \times 224$ \\
\hline Slice thickness & $5 \mathrm{~mm}$ & $5 \mathrm{~mm}$ \\
\hline No. of slices & 5 & 5 \\
\hline
\end{tabular}

Table 1. Acquisition parameters of the fast field-echo multi-shot EPI sequence used for fast $\mathrm{T} 2 *$ mapping of the lumbar bone marrow at $1.5 \mathrm{~T}$ 
Estimation of the $\mathrm{T}^{*}$ relaxation time was performed on one manually-defined region drawn on the entire L2 vertebral body as shown in Fig. 4. The multi-shot EPI sequence produced T2* maps with mean values comparable with previous data obtained with conventional sequences (Fig. 5). The mean T2* measured in the sagittal plane $(14.2 \pm 3.9 \mathrm{~ms})$ was slightly lower than that measured in the axial plane $(14.7 \pm 3.9 \mathrm{~ms})$ but no statistically significant difference was observed $(P<0.05)$.

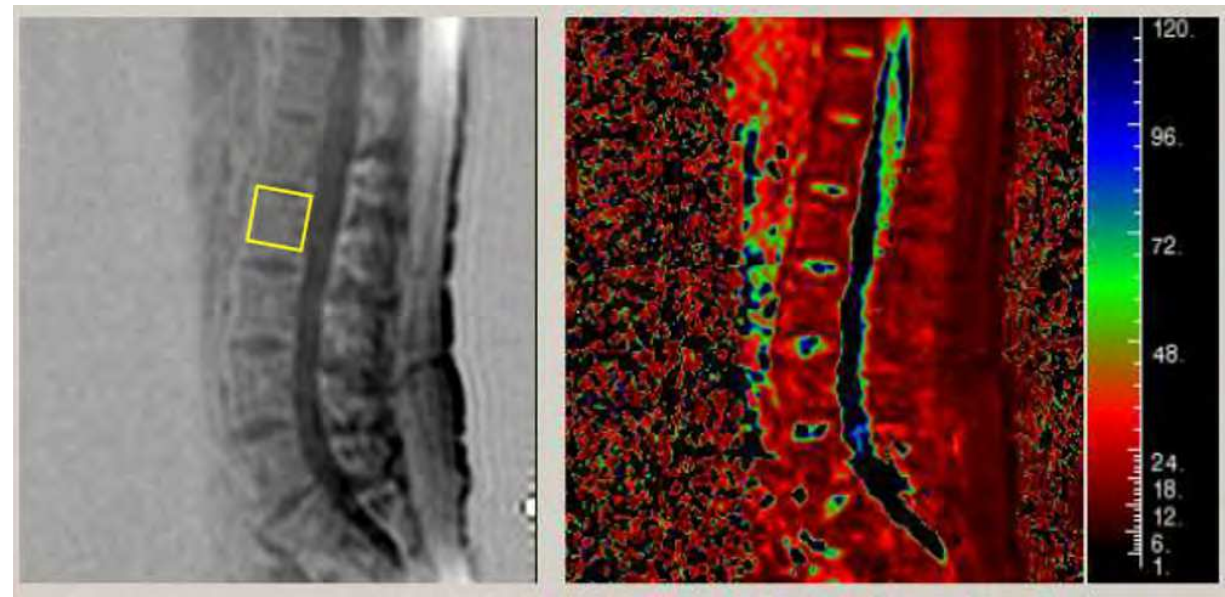

(a)
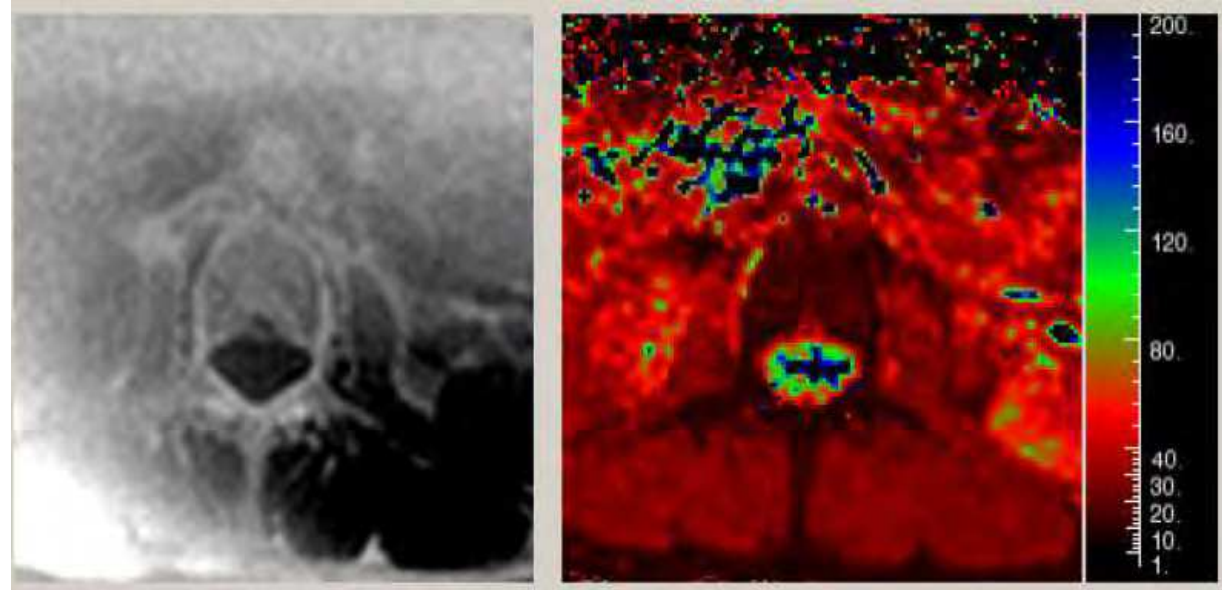

(b)

Fig. 4. T2* maps of the central slice of the L2 vertebra generated from sagittal (a) and axial (b) images by means of a monoexponential fitting algorithm as described by Dahnke \& Schaeffter (2005). The mean T2* was measured over the entire vertebral body excluding the cortical bone 

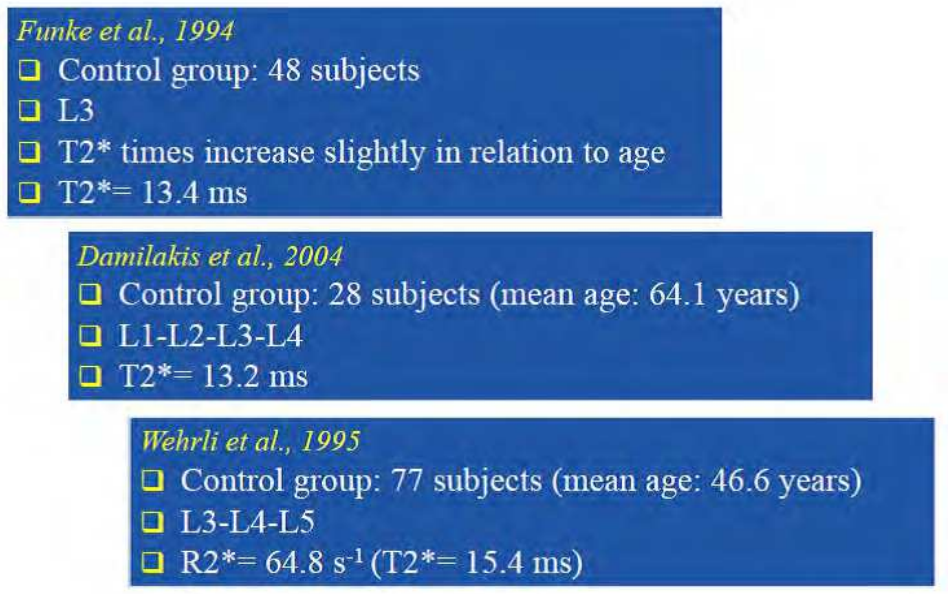

Fig. 5. Overview of the $\mathrm{T}^{*}$ data previously obtained with conventional sequences

These results indicate that fast $\mathrm{T}^{*}$ mapping of the lumbar bone marrow is feasible on a $1.5 \mathrm{~T}$ scanner. However, further studies are required to investigate the full potential of the proposed approach in the clinical evaluation of osteoporosis.

\section{MRI of articular cartilage}

Articular cartilage, is one of the types of hyaline cartilage that persists throughout adult life. Basically, it comprises chondrocytes incorporated in an extracellular matrix composed mainly of water, collagen II fibrils and proteoglycans (Seibel et al., 2004). Despite its simple appearance, this tissue hides various modifications in respect of the original cartilage that make it a singular structure. The articular cartilage is, in fact, stratified and classically, four distinct layers are described from the surface to the interior: tangential, transitional, radial and calcified, respectively (Fig. 6). Both morphological and biochemical information can be obtained by MRI, which is probably the most accurate imaging modality in evaluating the state of hyaline cartilage (Disler et al., 2000; Cova \& Toffanin, 2002). Apart from clinical MRI protocols that depict cartilage morphology, there is a growing interest in developing quantitative MRI approaches that are sensitive to its early structural changes (Burstein et al., 2000; Mosher \& Dardzinski, 2004).

Over the past years, quantification of the human articular cartilage has been performed using T1, T1 $\rho$ and T2 relaxation time constants as well as the magnetization transfer ratio (Toffanin et al., 2001; Menezes et al., 2004; Wheaton et al., 2005). One of the magnetic parameter that is currently evaluated for studying cartilage damage is the transverse relaxation time (T2), whose relaxation mechanism results dominated by the dipolar interaction between water molecules and collagen (Mlynárik et al., 2004) In this regard, dual-echo or multi-echo sequences are typically employed for quantitative T2 mapping. Nonetheless, faster quantitative MRI techniques are required in order to allow the introduction of T2 mapping in routine clinical protocols. 


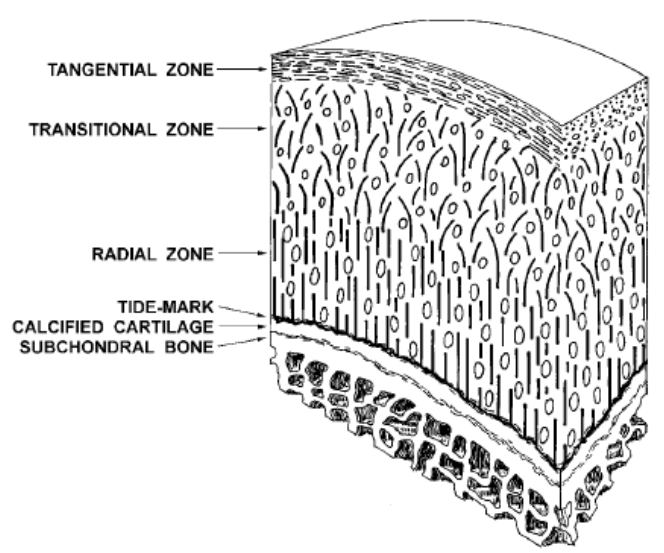

Fig. 6. Histological zones in hyaline cartilage. Collagen fibres are parallel to the surface in the superficial (tangential) zone, curved in the intermediate (transitional) zone and perpendicular to subchondral bone in the deep (radial) zone (From Cova \& Toffanin, 2002. Reprinted with permission).

\subsection{Fast T2 mapping of the patellar articular cartilage}

Recently, we have devoted particular attention to optimising specific quantitative MRI protocols for the fast T2 mapping of knee cartilage. The focus was on the gradient- and spinecho (GRASE) sequence able to produce a set of T2-weighted images in less than 2 minutes. Also this research study was conducted on a commercial clinical 1.5 T MRI scanner located in the Department of Radiology of the Cattinara Hospital at the University of Trieste. The feasibility of the proposed approach was assessed on 35 patients (21 males and 14 females) with moderate degree of patellar osteoarthritis. (Quaia et al., 2008).

For each patient, transverse GRASE and TSE images of patellar cartilage were acquired using the scan protocols summarised in Table 2.

\begin{tabular}{|l|c|c|}
\hline & GRASE & TSE \\
\hline TR & $3,000 \mathrm{~ms}$ & $3,000 \mathrm{~ms}$ \\
\hline $\mathrm{TE}_{\min }$ & $15 \mathrm{~ms}$ & $15 \mathrm{~ms}$ \\
\hline $\mathrm{TE}_{\max }$ & $120 \mathrm{~ms}$ & $120 \mathrm{~ms}$ \\
\hline EPI factor & 3 & - \\
\hline Turbo factor & 8 & 8 \\
\hline FOV & $80 \mathrm{~mm} \times 80 \mathrm{~mm}$ & $80 \mathrm{~mm} \times 80 \mathrm{~mm}$ \\
\hline Matrix & $128 \times 128$ & $128 \times 128$ \\
\hline Slice thickness & $3 \mathrm{~mm}$ & $3 \mathrm{~mm}$ \\
\hline No. of slices & 10 & 10 \\
\hline Total scan time & $1 \mathrm{~min} 51 \mathrm{~s}$ & $5 \mathrm{~min} 52 \mathrm{~s}$ \\
\hline
\end{tabular}

Table 2. Acquisition parameters of the GRASE and TSE sequences used for fast T2 mapping of the patellar articular cartilage at $1.5 \mathrm{~T}$ 

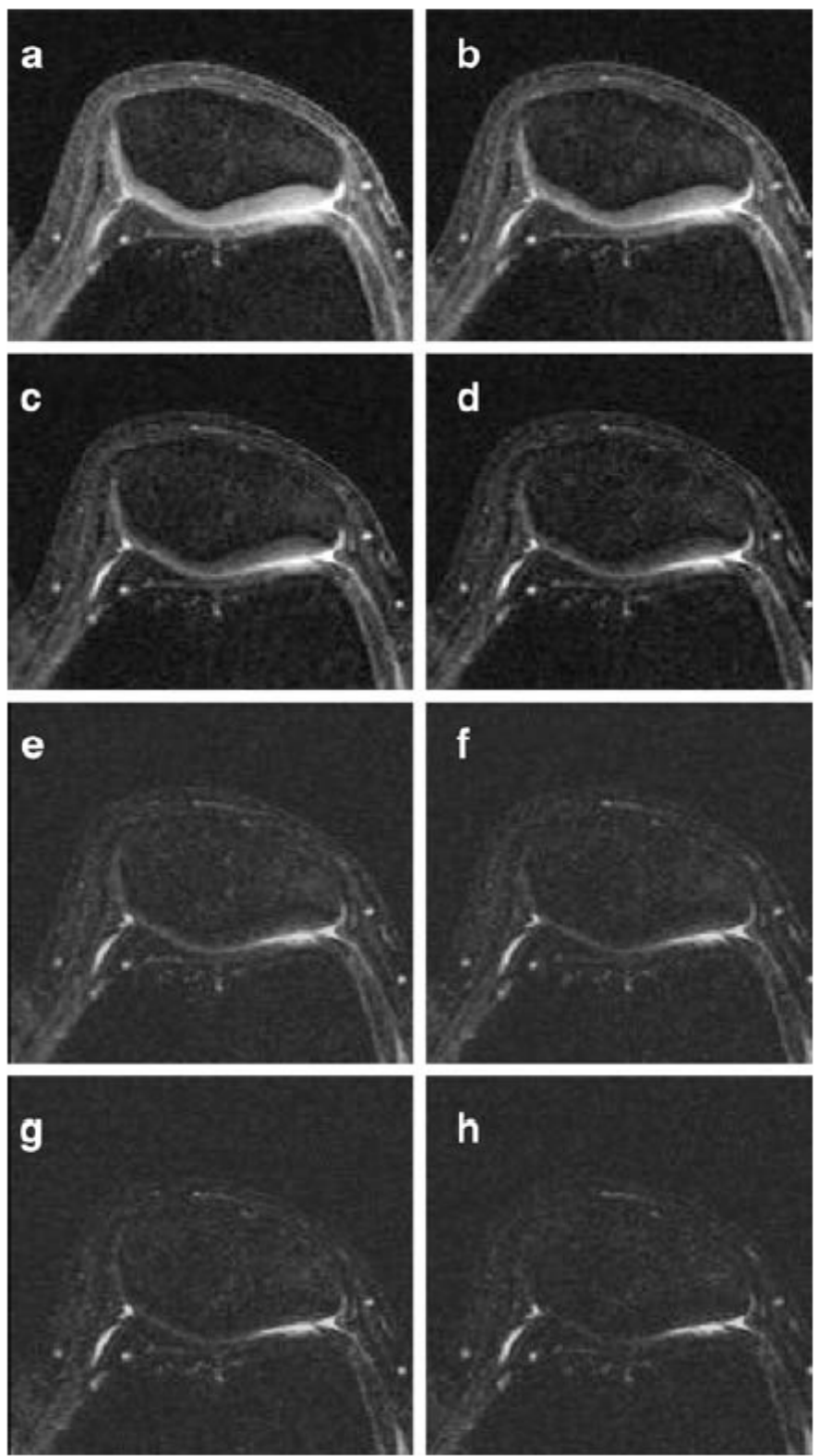

Fig. 7. a-h. Axial GRASE images of patella cartilage (TR/TE $\min _{-}-\mathrm{TE}_{\max }: 3,000 / 15-120 \mathrm{~ms}$ ); a: $15 \mathrm{~ms}$, b: $30 \mathrm{~ms}$, c: $45 \mathrm{~ms}$, d: $60 \mathrm{~ms}$, e: $75 \mathrm{~ms}$, f: $90 \mathrm{~ms}$, g: $105 \mathrm{~ms}$, h: $120 \mathrm{~ms}$. There is a clear decay in the signal intensity of the patellar articular cartilage at longer TE, which can be visually observed. The T2 relaxation time constant was calculated from a linear least-square fit to the logarithm of the image intensity data (From Quaia et al., 2008. Reprinted with permission). 
Estimation of T2 was performed on one manually-defined region drawn on the entire patella cartilage as shown in Fig. 8.

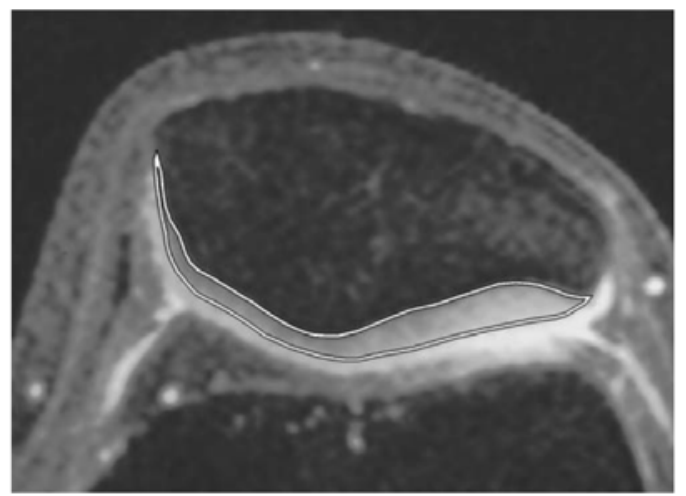

Fig. 8. Axial GRASE image of patella cartilage (TR/TE: 3,000/15 ms). A manually defined ROI is drawn on the entire patellar articular cartilage for the quantification of the global T2 relaxation time. The ROI includes the entire cartilage, encompassing both the deep cartilage close to the subchondral bone and the superficial cartilage, and the edges of the joint surface (From Quaia et al., 2008. Reprinted with permission).

\begin{tabular}{|c|c|c|c|}
\hline Patient No. & GRASE & TSE & Arthroscopic grading \\
\hline 1 & $44.0 \pm 10.0$ & $43.5 \pm 8.0$ & $2 \mathrm{~B}$ \\
\hline 2 & $48.3 \pm 8.0$ & $47.2 \pm 6.0$ & $2 \mathrm{~B}$ \\
\hline 3 & $25.5 \pm 9.0$ & $26.3 \pm 7.5$ & 0 \\
\hline 4 & $38.1 \pm 7.0$ & $38.4 \pm 6.0$ & 1 \\
\hline 7 & $45.2 \pm 6.0$ & $46.3 \pm 7.0$ & $3 \mathrm{~A}$ \\
\hline 12 & $30.5 \pm 8.0$ & $31.0 \pm 6.0$ & $2 \mathrm{~A}$ \\
\hline 18 & $35.5 \pm 8.0$ & $37.2 \pm 8.0$ & 1 \\
\hline 20 & $39.2 \pm 7.0$ & $38.5 \pm 6.0$ & $2 \mathrm{~A}$ \\
\hline 27 & $28.5 \pm 6.0$ & $29.0 \pm 9.0$ & 0 \\
\hline 28 & $58.2 \pm 6.0$ & $57.2 \pm 11.0$ & $3 \mathrm{~A}$ \\
\hline 35 & $61.2 \pm 11.0$ & $64.3 \pm 10.0$ & $3 \mathrm{~B}$ \\
\hline
\end{tabular}

Table 3. Mean T2 values for patellar articular cartilage obtained from the GRASE and TSE images of the patellar articular cartilage of selected patients together with the corresponding arthroscopic grading1.

\footnotetext{
${ }^{1}$ Osteoarthritis from anteroposterior radiographs was graded according to the Kellgren-Lawrence scoring system $(0=$ no osteoarthritic features; $1=$ minute osteophytes of doubtful importance; $2=$ definite osteophytes without reduction of the joint space; $3=$ reduction of the joint space; $4=$ greatly reduced joint space and sclerosis of the subchondral bone) (Kellgren \& Lawrence, 1957).
} 
In our series the GRASE sequence provided T2 values slightly lower than those obtained by the TSE sequence in most patients. This may relate to $\mathrm{T}^{*}$ decay present in the GRASE sequence. Indeed, a potential drawback of the GRASE sequence is the possible presence of artefacts due to the echo-planar imaging readout module that may lead to pronounced chemical shift, image distortion, and signal loss from magnetic field inhomogeneity. Nonetheless, differences in T2 values within deep and superficial cartilage were in line with those observed with other MR sequences (Van Breuseghem et al., 2004). They are related to cartilage T2 anisotropy determined by the different direction of the collagen fibres in the superficial and deep cartilage with respect to the static magnetic field (Mosher et al., 2001).

\section{Conclusion}

The above MRI methods can drastically reduce the scan time for measuring the spatial distribution of specific relaxation parameters in bone and cartilage. They may become more widely adopted, being applied either with other imaging techniques or in isolation, to better evaluate skeletal disorders, identify early tissue degeneration, tailor therapeutic interventions and follow treatment response.

\section{Acknowledgment}

This work was supported in part by a grant from the Italian Ministry for Education, University and Research (5 per mille 2009).

\section{References}

Abragam, A. (1983). The Principles of Nuclear Magnetism (2nd edition), Oxford University Press, ISBN 978-0-19-852014-6, New York

Apprich, S.; Welsch, G.H.; Mamisch, T.C.; Szomolányi, P.; Mayerhoefer, M.; Pinker, K. \& Trattnig, S. (2010). Detection of degenerative cartilage disease: comparison of highresolution morphological $\mathrm{MR}$ and quantitative $\mathrm{T} 2$ mapping at 3.0 Tesla. Osteoarthritis and Cartilage, Vol.18, No.9, pp. 1211-1217, ISSN 1063-4584

Burstein, D.; Bashir, A. \& Gray, M.L. (2000). MRI techniques in early stages of cartilage disease. Investigative Radiology, Vol.35, No.10, pp. 622-638, ISSN 0020-9996

Chung, H.; Wehrli, F.W.; Williams, J.L. \& Kugelmass, S.D. (1993). Relationship between NMR transverse relaxation, trabecular bone architecture, and strength. Proceedings of the National Academy of Sciences U.S.A., Vol.90, No.21, pp. 10250-10254, ISSN 0027-8424

Cova, M. \& Toffanin, R. (2002). MR microscopy of hyaline cartilage: current status. European Radiology, Vol.12, No.4, pp. 814-823, ISSN 0938-7994

Dahnke, H. \& Schaeffter, T. (2005). Limits of detection of SPIO at $3.0 \mathrm{~T}$ using T2* relaxometry. Magnetic Resonance in Medicine, Vol.53, No.5, pp 1202-1206, ISSN 0740-3194

Damilakis, J.; Maris, T.; Papadokostakis, G.; Sideri, L. \& Gourtsoyiannis, N. (2004). Discriminatory ability of magnetic resonance $\mathrm{T} 2 *$ measurements in a sample of postmenopausal women with low-energy fractures. A comparison with phalangeal speed of sound and dual X-ray absorptiometry. Investigative Radiology, Vol.39, No.11, pp. 706-712, ISSN 0020-9996 
David-Vaudey, E.; Ghosh, S.; Ries, M. \& Majumdar, S. (2004). T2 relaxation time measurements in osteoarthritis. Magnetic Resonance Imaging, Vol.22, No.5, pp. 673682, ISSN 0730-725X

Davis, C.A.; Genant, H.K. \& Dunham, J.S. (1986). The effects of bone on proton NMR relaxation times surrounding liquids. Investigative Radiology, Vol.21, No.6, pp. 472477, ISSN 0033-8419

Disler, D.G.; Recht, M.P. \& McCauley, T.R. (2000). MR imaging of articular cartilage. Skeletal Radiology, Vol.29, No.7, pp. 367-377, ISSN 0364-2348

Dunn, T.C.; Lu, Y.; Jin, H.; Ries, M.D. \& Majumdar, S. (2004). T2 relaxation time of cartilage at MR imaging: comparison with severity of knee osteoarthritis. Radiology, Vol.232, No.2, pp. 592-598, ISSN 0033-8419

Feinberg, D.A. \& Oshio, K. (1991). GRASE (Gradient- and spin-echo) MR imaging: a new fast clinical imaging technique. Radiology, Vol.181, No.2, pp. 597-602, ISSN 0033-8419

Funke, M.; Bruhn, H.; Vosshenrich, R.; Rudolph, O. \& Grabbe, E. (1994). The determination of the T2* relaxation time for characterizing trabecular bone. Rofo-Fortschritte auf dem Gebiet der Röntgenstrahlen und der Bildgebenden Verfahren, Vol.161, No.1, pp. 5863, ISSN 1438-9029

Grampp, S.; Majumdar, S.; Jergas, M. Lang P, Gies, A. \& Genant, H.K. (1995). MRI of bone marrow in the distal radius: in vivo precision of effective transverse relaxation times. European Radiology, Vol.5, No.1, pp. 43-48, ISSN 0938-7994

Guglielmi, G.; Biccari, N.; Mangano, F. \& Toffanin, R. (2010). 3 T magnetic resonance imaging of the musculoskeletal system. La Radiologia Medica, Vol.115, No.4, pp. 571584, ISSN: 0033-8362

Hannan, H.L.; Magaziner, J.; Wang, J.J.; Eastwood, E.A.; Silberzweig, S.B.; Gilbert, M.; Morrison, R.S.; McLaughlin, M.A.; Orosz, G.M. \& Siu, A.L. (2001). Mortality and locomotion 6 months after hospitalization for hip fracture: risk factors and riskadjusted hospital outcomes. JAMA, Vol.285, No.21, pp. 2736-2742, ISSN 00987484

Hennig, J.; Nauerth, A. \& Friedburg, H. (1986). RARE imaging: a fast imaging method for clinical MR. Magnetic Resonance in Medicine, Vol.3, No.6, pp. 823-833, ISSN 07403194

Jergas, M.D.; Majumdar, S.; Keyak, J.H.; Lee, I.Y.; Newitt, D.C.; Grampp, S.; Skinner, H.B. \& Genant, H.K. (1995). Relationship between young modulus of elasticity, ash density, and MRI derived effective transverse relaxation $\mathrm{T}^{*}$ in tibial specimens. Journal of Computed Assisted Tomography, Vol.19, No.3, pp. 472-479, ISSN 0363-8715

Kellgren, J. \& Lawrence, J. (1957). Radiological assessment of osteoarthritis. Annals of the Rheumatic Diseases Vol.16, No.4, pp. 494-502, ISSN 00034967

Link, T.; Majumdar, S.; Augat, P.; Lin, J.C.; Newitt, D.; Lane, N.E. \& Genant, H.K. (1998). Proximal femur: assessment for osteoporosis with $\mathrm{T}^{*}$ decay characteristics at MR imaging. Radiology, Vol.209, No.2, pp. 531-536, ISSN 0033-8419

Majumdar, S. (2008). Magnetic resonance imaging for osteoporosis. Skeletal Radiology, Vol.37, No.2, pp. 95-97, ISSN 0364-2348

Mansfield, P. (1977). Multi-planar imaging formation using NMR spin-echo. Journal of Physics C: Solid State Physics, Vol.10, pp. L55-L58, ISSN 0022-3719

Mehlkopf, A.F.; van der Meulen, P. \& Smidt, J. (1984). A multiple-echo and multiple-shot sequence for fast NMR-Fourier imaging. Magnetic Resonance in Medicine, Vol.1, No.2, pp. 295-297, ISSN 0740-3194 
Menezes, N.M.; Grey, M.L.; Hartke, J.R. \& Burstein, D. (2004). T2 and T1(rho) MRI in articular cartilage systems. Magnetic Resonance in Medicine, Vol.51, No.3, pp. 503509, ISSN 0740-3194

Mlynárik, V.; Szomolányi, P.; Toffanin, R.; Vittur, F. \& Trattnig, S. (2004). Transverse relaxation mechanisms in articular cartilage. Journal of Magnetic Resonance, Vol.169, No.2, pp. 300-307, ISSN 1090-7807

Mosher, T.J.; Smith, H.; Dardzinski, B.J.; Schmithorst, V.J. \& Smith, M.B. (2001). MR imaging and T2 mapping of femoral cartilage: in vivo determination of the magic angle effect. AJR American Journal of Roentgenology, Vol.177, No.3, pp. 665-669, ISSN 0361-803X

Mosher, T.J. \& Dardzinski, B.J. (2004). Cartilage MRI T2 relaxation time mapping: overview and applications. Seminars in Musculoskeletal Radiology, Vol.8, No.4, pp. 355-368, ISSN 1089-7860

Mugler, J.P. III. (2006). Basic principles. In: Clinical Magnetic Resonance Imaging, Edelman, R.R.; Hesselink, J.R.; Zlatkin, M.B.; Crues, J.V., Editors, pp. 23-57, Elsevier, ISBN 978-0721603063, Philadelphia

Oshio, K. \& Feinberg, D.A. (1991). GRASE (Gradient- and Spin-Echo) imaging: a novel fast MRI technique. Magnetic Resonance in Medicine, Vol.20, No.2, pp. 344-349, ISSN 0740-3194

Quaia, E.; Toffanin, R.; Guglielmi, G.; Ukmar, M.; Rossi, A.; Martinelli, B. \& Cova, M.A. (2008). T2 mapping of the patellar articular cartilage with gradient and spin-echo magnetic resonance imaging at $1.5 \mathrm{~T}$ : validation and initial clinical experience in patients with osteoarthritis. Skeletal Radiology, Vol.37, No.6, pp. 511-517, ISSN 0364-2348

Rosenthal, H.; Thulborn, K.R.; Rosenthal, D.I.; Kim, S.H. \& Rosen, B.R. (1990). Magnetic susceptibility effects of trabecular bone on magnetic resonance bone marrow imaging. Investigative Radiology, Vol.25; No.2, pp. 173-178, ISSN 0020-9996

Seibel, M.J.; Robins, S.P. \& Bilezikian, J.P. (Eds.). (2004). Dynamics of Bone and Cartilage Metabolism, Academic Press, ISBN 978-0-12-088562-6, San Diego, USA

Theis, K.A.; Murphy, L.; Hootman, J.M.; Helmick, C.G. \& Yelin, E. (2007). Prevalence and correlates of arthritis-attributable work limitation in the US population among persons ages 18-64: 2002 National Health Interview Survey Data. Arthritis $\mathcal{E}$ Rheumatism, Vol.57, No.3, pp. 355-363, ISSN 1529-0131

Toffanin, R.; Mlynárik, V.; Russo, S.; Szomolányi, P.; Piras, A. \& Vittur, F. (2001). Proteoglycan depletion and MR parameters of articular cartilage. Archives of Biochemistry and Biophysics, Vol.390, No.2, pp. 235-242, ISSN 0003-9861

Toffanin, R.; Cadioli, M.; Scotti, G. \& Cova, M.A. (2006). Ultrafast T2* mapping of bone marrow at 1.5 Tesla and 3.0 Tesla. Proceedings of the 14th Annual Meeting of the International Society for Magnetic Resonance in Medicine, p. 3631, Seattle, Washington, USA, May 6-12, 2006, ISSN 1545-4428

Tsao, J. (2010). Ultrafast imaging: principles, pitfalls, solutions, and applications. Journal of Magnetic Resonance Imaging, Vol.32, No.2, pp. 252-266, ISSN 1053-1807

Van Breuseghem, I.; Bosmans, H.T.; Elst, L.V.; Maes, F.; Pans, S.D.; Brys, P.P.; Geusens, E.A. \& Marchal, G.J. (2004). T2 mapping of human femorotibial cartilage with turbo mixed MR imaging at 1.5 T: feasibility. Radiology, Vol.233, No.2, pp. 609-614, ISSN 0033-8419

Wasnich, R.D. (1999). Incidence Rates for Vertebral, Wrist \& Hip Fractures in Women after Age 50. In: Primer on the Metabolic Bone Diseases and Disorders of Mineral Metabolism. 
(4th edition), Murray, F.J., Editor, pp. 257-259, Lippincott, Williams \& Wilkins, ISBN 978-0781720380, Philadelphia

Wehrli, F.W.; Hilaire, L.; Fernández-Seara, M; Gomberg, B.R.; Song, H.K.; Zemel, B.; Loh, L. \& Snyder, P.J. (2002). Quantitative magnetic resonance imaging in the calcaneus and femur of women with varying degrees of osteopenia and vertebral deformity status. Journal of Bone Mineral Research, Vol.17, No.12, pp. 2265-2273, ISSN 0884-0431

Wehrli, F.W.; Ford, J.C. \& Haddad, J.G. (1995). Osteoporosis: clinical assessment wit quantitative MR imaging in diagnosis. Radiology, Vol.196, No.3, pp. 631-641, ISSN 0033-8419

Wehrli, F.W.; Song, H.K.; Saha, P.K. \& Wright, A.C. (2006). Quantitative MRI for the assessment of bone structure and function. NMR in Biomedicine, Vol.19, No.7, pp. 731-776, ISSN 0952-3480

Wheaton, A.J.; Dodge, G.R.; Borthakur, A.; Kneeland, J.B.; Schumacher, H.R. \& Reddy, R. (2005). Detection of changes in articular cartilage proteoglycan by T1(rho) magnetic resonance imaging. Journal of Orthopaedic Research, Vol.23, No.1, pp. 102-108, ISSN 0736-0266 


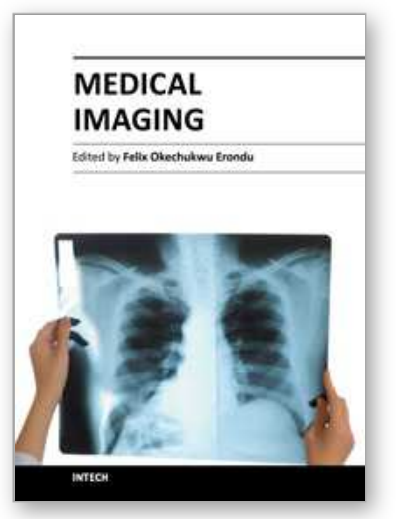

\author{
Medical Imaging \\ Edited by Dr. Okechukwu Felix Erondu
}

ISBN 978-953-307-774-1

Hard cover, 412 pages

Publisher InTech

Published online 22, December, 2011

Published in print edition December, 2011

What we know about and do with medical imaging has changed rapidly during the past decade, beginning with the basics, following with the breakthroughs, and moving on to the abstract. This book demonstrates the wider horizon that has become the mainstay of medical imaging sciences; capturing the concept of medical diagnosis, digital information management and research. It is an invaluable tool for radiologists and imaging specialists, physicists and researchers interested in various aspects of imaging.

\title{
How to reference
}

In order to correctly reference this scholarly work, feel free to copy and paste the following:

Renato Toffanin, Giuseppe Guglielmi and Maria A. Cova (2011). Fast MRI Methods for the Clinical Evaluation of Skeletal Disorders, Medical Imaging, Dr. Okechukwu Felix Erondu (Ed.), ISBN: 978-953-307-774-1, InTech, Available from: http://www.intechopen.com/books/medical-imaging/fast-mri-methods-for-the-clinical-evaluationof-skeletal-disorders

\section{INTECH}

open science | open minds

\section{InTech Europe}

University Campus STeP Ri

Slavka Krautzeka 83/A

51000 Rijeka, Croatia

Phone: +385 (51) 770447

Fax: +385 (51) 686166

www.intechopen.com

\section{InTech China}

Unit 405, Office Block, Hotel Equatorial Shanghai

No.65, Yan An Road (West), Shanghai, 200040, China

中国上海市延安西路65号上海国际贵都大饭店办公楼 405 单元

Phone: +86-21-62489820

Fax: $+86-21-62489821$ 
(C) 2011 The Author(s). Licensee IntechOpen. This is an open access article distributed under the terms of the Creative Commons Attribution 3.0 License, which permits unrestricted use, distribution, and reproduction in any medium, provided the original work is properly cited. 